\title{
Macroscopic quantum tunneling and retrapping processes in moderately damped $\mathrm{YBaCuO}$ Josephson junctions
}

\author{
D. Massarotti ${ }^{1,2}$, L. Longobardi ${ }^{3,2}$, L. Galletti ${ }^{1,2}$, D. Stornaiuolo ${ }^{2}$, \\ G. Rotoli ${ }^{3}$, and F. Tafuri ${ }^{3,2,1}$ \\ ${ }^{1}$ Dip. Scienze Fisiche, Universitá di Napoli Federico II, Monte S. Angelo via Cinthia, Napoli 80126, Italy \\ E-mail: dmassarotti@na.infn.it \\ ${ }^{2}$ CNR-SPIN, UOS Napoli, Monte S. Angelo via Cinthia, Napoli 80126, Italy \\ ${ }^{3}$ Dip. Ingegneria dell'Informazione, Seconda Universitá di Napoli, via Roma 29, Aversa (CE) 81031, Italy
}

Received November 1, 2012

\begin{abstract}
The moderately damped regime in a Josephson junction (JJ) is quite common in devices characterized by low critical currents and therefore by low Josephson energies. Measurements of switching current distribution (SCD) are a direct way of discriminating the phase dynamics also in the nontrivial case of moderate damping, which is going to be more and more common with advances in nanopatterning superconductors and in materials science finalized to build hybrid systems. We report on measurements of SCDs, both in thermal and quantum regime, on moderately damped $\mathrm{YBaCuO}$ grain boundary biepitaxial JJs. A direct transition from phase diffusion regime to macroscopic quantum tunnelling occurs at about $130 \mathrm{mK}$. The crossover to the quantum regime is tuned by the magnetic field and phase dynamics is described by a fully consistent set of junction parameters derived through numerical simulations.
\end{abstract}

PACS: 74.72.-h Cuprate superconductors;

85.25.Cp Josephson devices;

74.50.+r Tunneling phenomena; Josephson effects.

Keywords: phase dynamics, MQT, moderately damped regime, phase diffusion.

\section{Introduction}

In the last few years, studies on phase dynamics and macroscopic quantum phenomena have been extended to junctions composed of materials other than the traditional low critical temperature superconductors (LTS) [1-3] and to novel types of structures with unconventional barriers composed for instance of graphene sheets [4] or of grain boundaries [1]. Issues on macroscopic quantum phenomena on wire and nanostructures have been renewed by the advances in nanopatterning and in achieving high quality nanowires [5,6]. Progress in engineering new materials into junctions and in understanding and controlling the physics of interfaces may offer novel solutions for junctions of superior quality and complementary functionalities, and therefore may lead in the long run to improve also specific qubit performances [7]. Measurements of switching current distribution (SCD) have turned to be more and more standard tools to investigate phase dynamics in unconventional and hybrid systems and nanostructures. High critical temperature superconductors (HTS) are an example of unconventional systems, because of the $d$-wave order parameter symmetry and of the presence of low-energy quasiparticles. In HTS Josephson junctions (JJs) lowenergy quasiparticles are expected to induce higher levels of dissipation [8-10] when compared to LTS systems. A comparative study in systems so different and complementary is of great interest. HTS systems are characterized in suitable conditions by intermediate levels of dissipation, that make these junctions fall in the moderately damped regime (MDR). In LTS systems, MDR is more frequently the natural consequence of a reduction of the critical current $I_{c}$. Low $I_{c}$ are induced or by size reduction typically in the submicron regime [11-15] or by very low critical current density $J_{c}$ [16-18]. Lower critical currents result in lower Josephson energies. These devices are charac- 
terized by intermediate levels of dissipation and by phase diffusion (PD) phenomena [11,13-16, 18-21]. The low critical current $I_{c}$ limit seems to be characteristic also of all futuristic nanohybrids devices incorporating nanowires or unconventional barriers [18,22-24], and MDR is intrinsically more common than it could be expected. In this work we report on measurements of SCDs on moderately damped $\mathrm{YBa}_{2} \mathrm{Cu}_{3} \mathrm{O}_{7-x}$ grain boundary (GB) biepitaxial JJs down to the quantum regime [25]. The capability to engineer junctions on different substrates offers the opportunity to disentangle the role of the shell circuit in the phase dynamics and thus a tuning of the capacitive effects in HTS GB biepitaxial JJs. Experimental data are supported by Monte Carlo simulations of the phase dynamics, in a wide range of temperatures and dissipation levels. This allows to address relevant issues on how to quantify dissipation in the MDR and a partial reconstruction of a phase diagram as guideline for a wide range of moderately damped systems.

\section{Resistively and capacitively shunted junction model and phase dynamics}

The dynamics of a current-biased JJ is governed by the phase difference $\varphi$ of the order parameter across the junction. In the framework of the resistively and capacitively shunted junction (RCSJ) model [26], the phase dynamics is equivalent to the motion of a particle in a washboard potential $U=-E_{J}\left(\cos \varphi+I / I_{c 0}\right)$ (see Fig. 1):

$$
\left(\frac{\phi_{0}}{2 \pi}\right)^{2} C \frac{\partial^{2} \varphi}{\partial t^{2}}+\left(\frac{\phi_{0}}{2 \pi}\right)^{2} \frac{1}{R} \frac{\partial \varphi}{\partial t}+\frac{\partial U}{\partial \varphi}=0,
$$

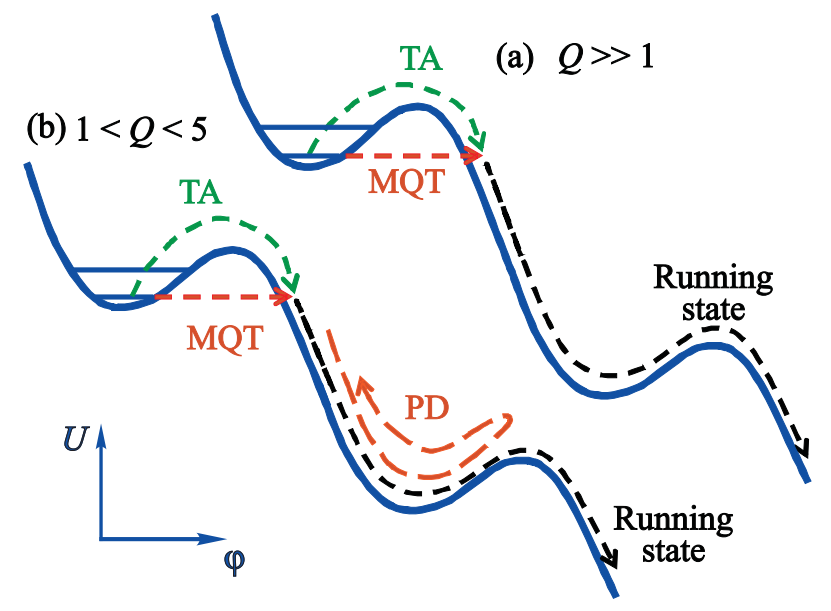

Fig. 1. (Color online) Phase dynamics in the tilted periodic potential $U$ : thermal (TA) (green dashed line) and quantum (MQT) (red dashed line) escapes are shown in the ideal case of very low dissipation $(Q>>1)$ (a). In the MDR $(1<Q<5)$ the MQT rate is significantly affected by dissipation (see Eq. 3) and retrapping processes in subsequent potential wells become relevant, giving rise to the PD regime (b). $\phi_{0}$ is the flux quantum, $I_{c 0}$ represents the junction critical current in absence of thermal fluctuations and the term involving the capacitance $C$ represents the mass of the particle respectively. $E_{J}=I_{c 0} \phi_{0} / 2 \pi$ is the Josephson energy and the tilt of the washboard potential is given by the bias current $I$.

The strength of the friction can be expressed through the junction quality factor $Q=\omega_{p} R C$, where $\omega_{p}=$ $=\sqrt{2 e I_{c 0} / \hbar C}$ is the plasma frequency. When the bias current is ramped from $I=0$ to $I<I_{c 0}$, the junction is in the zero voltage state in absence of thermal and quantum fluctuations and the particle is confined to one of the potential wells, where it oscillates at the plasma frequency. When ramping the bias current $I$, the tilt of the energy potential increases and the height $\Delta U(I)=(4 \sqrt{2} / 3) E_{J}\left(1-I / I_{c 0}\right)^{3 / 2}$ of the energy barrier between consecutive wells decreases. Due to effects of thermal fluctuations and quantum tunneling the junction may switch to the finite voltage state for values of $I<I_{c 0}$. The relative weight of these two escape processes depends on the temperature of the system. For $k_{B} T>>\hbar \omega_{p}$ the escape process is dominated by thermal activation (TA) with a rate [27]:

$$
\Gamma_{T}(I)=a_{T} \frac{\omega_{p}(I)}{2 \pi} \exp \left(-\frac{\Delta U(I)}{k_{B} T}\right) .
$$

At low enough temperature the escape is dominated by macroscopic quantum tunneling (MQT) with a rate [28]:

$$
\Gamma_{q}(I)=a_{q} \frac{\omega_{p}(I)}{2 \pi} \exp \left[-\frac{\Delta U(I)}{\hbar \omega_{p}}\left(1+\frac{0.87}{Q}\right)\right] .
$$

Once the phase particle gets over a hill by fluctuations, it keeps running, provided that the damping is below some critical value. The escape from this metastable state corresponds to the appearance of a finite voltage across the junction and the particle runs down the washboard potential with a damping $Q^{-1}$. The transition to the running state (see Fig. 1(a)) only occurs if the kinetic energy gained by the phase particle running down the tilted washboard potential is not all dissipated, but enough energy remains to carry the phase over the next hill. This occurs if the junction is in the underdamped regime $(Q>>1)$ [29]. In the experiments of Ref. 29, a detailed experimental protocol has been established to prove the quantum behavior of the phase $\varphi$ across a JJ and its crossover to the thermal regime, used in most of later experiments. The relevant parameters of the junction and the dissipation level have been determined in situ in the thermal regime from measurements of resonant activation in the presence of microwaves. Such method still represents the most powerful way to characterize the dissipation level in the underdamped regime. In the moderately damped regime $(1<Q<5)$, following an event of escape the particle may travel down the potential for a few wells and then be retrapped in one of 
the following minima of the potential (Fig. 1(b)) [11,20]. The analytical expression for the retrapping rate is given by:

$$
\Gamma_{R}(I)=\omega_{p} \frac{I-I_{R}}{I_{c 0}}\left(\frac{E_{J}}{2 \pi k_{B} T}\right)^{1 / 2} \exp \left[-\frac{E_{J} Q^{2}}{2 k_{B} T}\left(\frac{I-I_{R}}{I_{c 0}}\right)^{2}\right],
$$

where $I_{R}=4 I_{c 0} / \pi Q$ is the retrapping current in absence of thermal fluctuations [30]. At low bias the process of escape and retrapping may occur multiple times generating diffusion of the phase until an increase of the tilt of the potential, due to a change in the bias current, raises the velocity of the particle and the transition to the running state occurs. This is known as PD regime [11,13-16,18-21]. The experimental observation of such regimes in a $\mathrm{JJ}$ is based on the measurement of the SCDs and the study of the behavior of its first and second momenta (the mean $\bar{I}$ and the width $\sigma$ ) as function of temperature. The resulting distribution of the switching probability $P(I)$ is used to compute the escape rate out of the zero-voltage state as a function of the bias current $I[31]$ :

$$
\Gamma(I)=\frac{1}{\Delta I} \frac{d I}{d t} \ln \left(\frac{\sum_{i \geq I} P(I)}{\sum_{i \geq I+\Delta I} P(I)}\right),
$$

where $d I / d t$ is the current ramp rate and $\Delta I$ is the channel width of the analog-to-digital converter. In an underdamped junction $(Q>10)$ [29], below a crossover temperature $T_{\text {cr }}$ the escape process is due to MQT, marked by a temperature independent $\sigma$, while above $T_{\text {cr }}$ the process of escape is due to TA with a distinctive increase of $\sigma$ with temperature as $T^{2 / 3}$. In moderately damped junctions $[11,13-16,18-21]$ a transition from TA to PD regime occurs at a crossover temperature $T^{*}>T_{\mathrm{cr}} . T^{*}$ corresponds to a distinctive change in the sign of the temperature derivative of $\sigma$, with $d \sigma / d T>0$ for $T<T^{*}$ and $d \sigma / d T<0$ for $T>T^{*}$.

\section{Phase dynamics of $\mathrm{YBaCuO}$ grain boundary biepitaxial Josephson junctions}

The search of macroscopic quantum effects has become feasible in HTS systems once high-quality JJs with significant hysteresis in the current-voltage $(I V)$ characteristics were available. MQT and energy level quantization were first observed in $\mathrm{YBa}_{2} \mathrm{Cu}_{3} \mathrm{O}_{7-x}$ GB biepitaxial JJ [1]. A specific feature of these structures is the use of a [110]-oriented $\mathrm{CeO}_{2}$ buffer layer, deposited on [110] $\mathrm{SrTiO}_{3}$ (STO) substrates. YBCO grows along the [001] direction on the $\mathrm{CeO}_{2}$ seed layer, while it grows along the [103]/[013] direction on STO substrates. In the experiment of Ref. 1, the measurements of SCDs as a function of temperature substantially follow what commonly measured on LTS JJs, with a saturation of the measured $\sigma$ below $50 \mathrm{mK}$, which corresponds to the crossover temperature $T_{\mathrm{cr}}$ from the thermal to the MQT regime.

The capacitance $C$ plays a role of primary importance in setting phase dynamics, as evident from Eq. (1) [26]. In recent studies on PD $[19,32]$, the possibility to vary $C$ on a broader scale was relevant to tune the transition temperature $T^{*}$. Such studies have the goal to "avoid" the PD regime by extending the TA regime to higher temperatures; a solid method to characterize the dissipation level in MDR is missing. In HTS JJs a complete understanding of the capacitive effects is still lacking. In STO based biepitaxial devices, the $\mathrm{JJ}$ is embedded in a shell $L C$ circuit, which originates from a large kinetic inductance, due to the current flow in the $c$-axis direction, and from a capacitive element in parallel to the junction, due to the large stray capacitance of the STO substrate [33]. In this work we have engineered junctions on $\left(\mathrm{La}_{0.3} \mathrm{Sr}_{0.7}\right)\left(\mathrm{Al}_{0.65} \mathrm{Ta}_{0.35}\right) \mathrm{O}_{3}$ (LSAT) rather than STO substrates. The new design fully responds to the task of reducing stray capacitances. The resulting device is a relevant term of comparison to search the origin of the capacitive effect in GB JJs, and is more representative of the intrinsic nature of the GB [25,34]. Specific capacitances are on average one order of magnitude lower than those measured on STO-based devices $[1,34]$. To study the escape rates of $\mathrm{YBaCuO}$ GB JJs we have thermally anchored the sample to the mixing chamber of a ${ }^{3} \mathrm{He} /{ }^{4} \mathrm{He}$ Oxford dilution refrigerator and performed measurements of the junction switching current probability. A full description of the apparatus is discussed in detail elsewhere [16]. Filtering is guaranteed by a room temperature electromagnetic interference filter stage followed by low pass $R C$ filters with a cut-off frequency of $1.6 \mathrm{MHz}$ anchored at $1.5 \mathrm{~K}$, and by a combination of copper powder and twisted pair filters thermally anchored at the mixing chamber of the dilution refrigerator. The bias current of the junction is ramped at a constant sweep rate $d I / d t=20 \mathrm{MA} / \mathrm{s}$ and at least $10^{4}$ switching events have been recorded using a standard technique. We report data for two different samples with interface orientations of $50^{\circ}$ for sample A and $75^{\circ}$ for sample B respectively. These angles determine robust overlap of the $d$-wave lobes on both sides of the junctions [10,34,35].

SCDs collected over a wide range of temperatures on sample A are reported in Fig. 2(a) and 2(b) for two different values of the magnetic field $(H=0$ and $H=12 \mathrm{G}$ respectively). The temperature dependence of the width $\sigma$ of the SCD curves measured on sample A and B is shown in Fig. 3(a). Our data are characterized by two distinct regimes. In Fig. 2(a), when increasing the temperature above $140 \mathrm{mK}$ the switching current hystograms shrink rather than broaden, which corresponds to the negative temperature derivative of $\sigma$ in Fig. 3(a). This behavior is consistent with a diffusive motion due to multiple escape and retrapping processes in the potential wells. The $\sigma$ dependence 

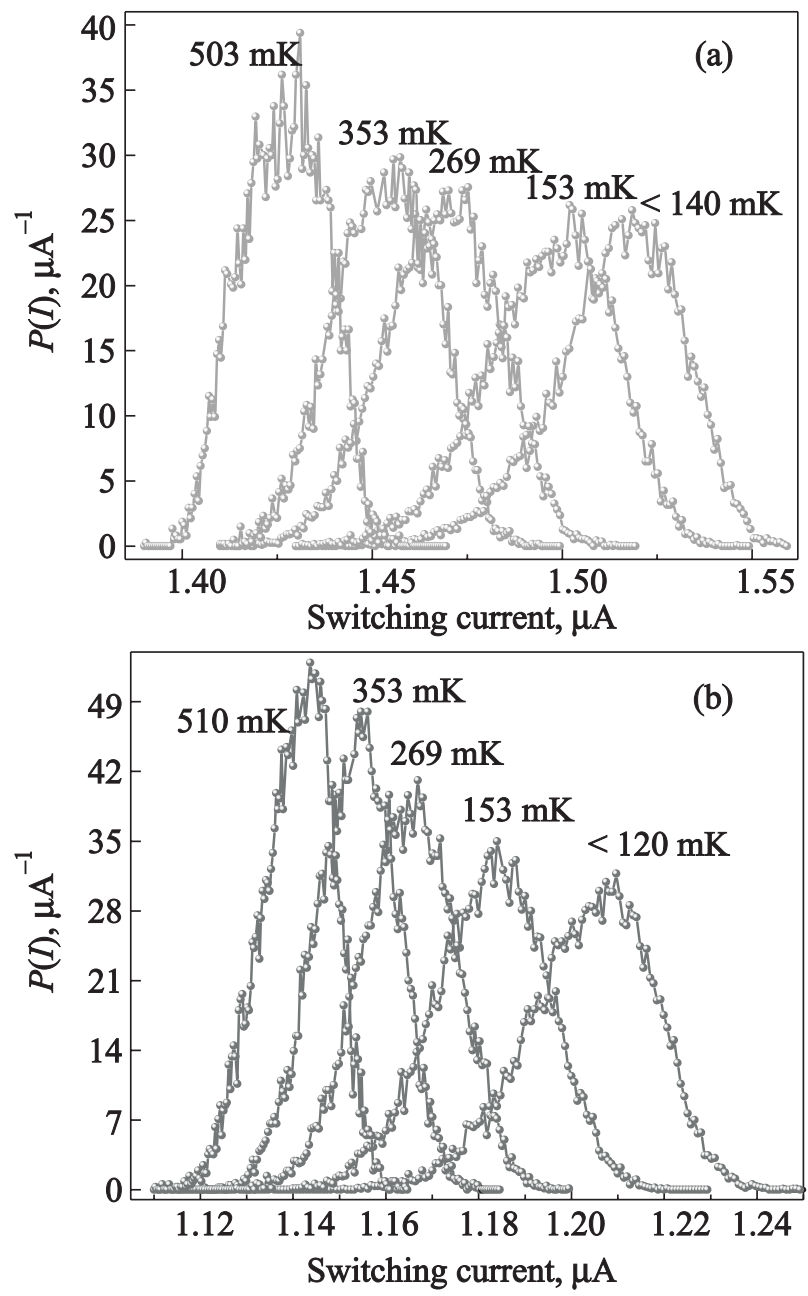

Fig. 2. Switching current hystograms as function of temperature measured on sample A for $H=0$ (a) and $H=12 \mathrm{G}$ (b), respectively. Above the crossover temperature $T_{\mathrm{cr}}\left(T_{\mathrm{cr}} \simeq 140 \mathrm{mK}\right.$ in (a) and $T_{\mathrm{cr}} \simeq 120 \mathrm{mK}$ in (b)), the hystograms shrink rather than broaden and become more symmetric. These are signatures of effectiveness of retrapping processes.

has been fitted through Monte Carlo simulations [20,25] with a damping factor $Q=1.3$ (light grey line in Fig. 3(a)). The dissipative essence of the quality factor $Q$ strongly depends on the value of the effective frequency dependent resistance $R(\omega)$ and of shunting capacitance $C$, which are in turn determined by several interplaying effects, such as circuit impedance, subgap resistance and stray capacitance [11]. Since these parameters are not easily accessible, a reliable way able to estimate the quality factor $Q$ in the MDR is of great interest. Below $140 \mathrm{mK}$, hystograms overlap and $\sigma$ saturates, which is a typical signature of a quantum activation regime. In analogy to what commonly done to prove MQT in underdamped junctions [29], we use the magnetic field to tune in situ the junction parameters and the quantum crossover temperature $T_{\text {cr }}$ to unambiguously prove MQT as source of the saturation of $\sigma$ below $T_{\mathrm{cr}}$. In the inset of Fig. 3(a) we report the temperature dependence of $\sigma$ measured for sample $A$ at the two different magnetic
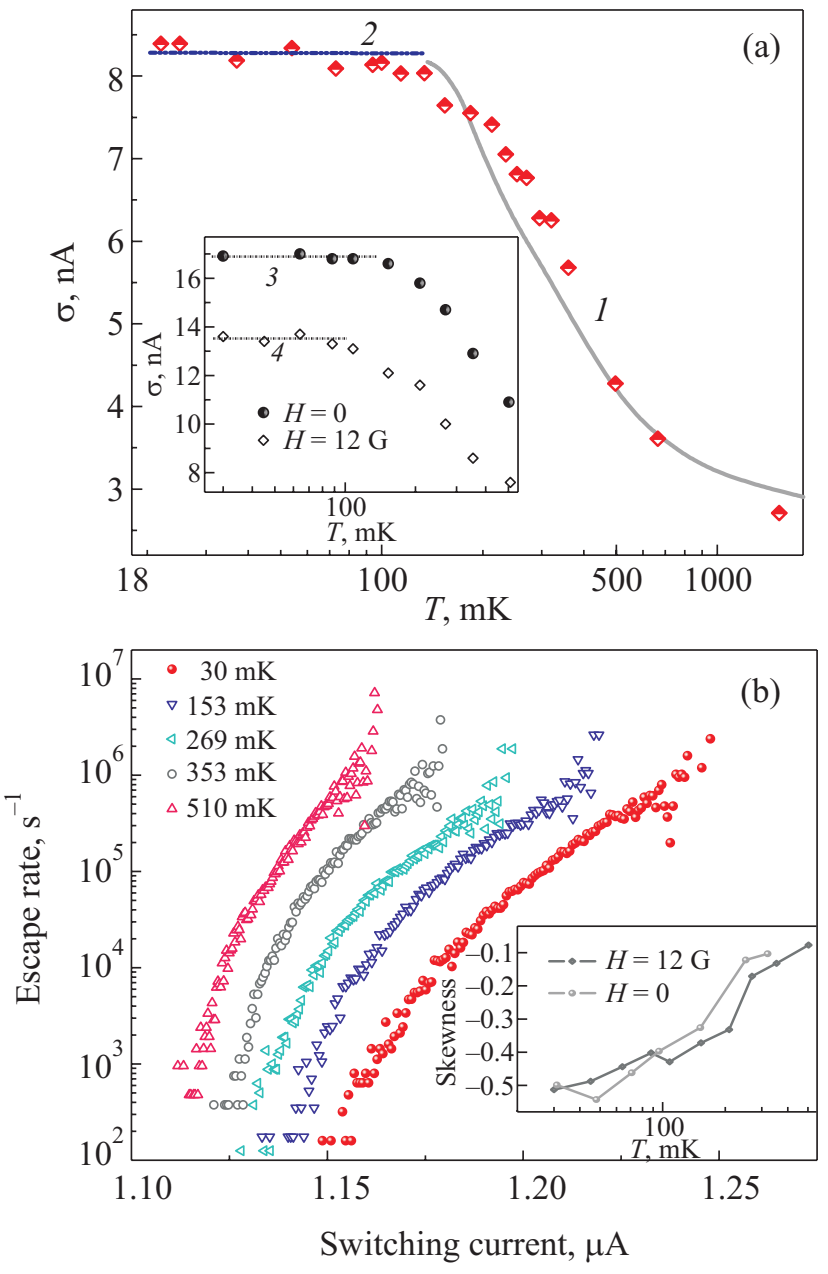

Fig. 3. (Color online) Thermal behavior of $\sigma$ of the SCDs measured on sample B. The light grey solid line (1) is the result of Monte Carlo simulations in the diffusive regime with a quality factor $Q=1.3$, while the blue dashed line (2) indicates the saturation of $\sigma$ below $T_{\mathrm{cr}}$. The inset shows temperature dependent data for sample A at $H=0$ and $H=12 \mathrm{G}$. The black dashed lines $(3,4)$ indicate the average values of $\sigma$ in MQT regime and the value of $T_{\mathrm{cr}}$, which are both reduced by the magnetic field (a). Experimental escape rates $\Gamma$ of sample A are shown as function of the switching current. In the inset, thermal behavior of the skewness $\gamma$ is reported for $H=0$ and $H=12 \mathrm{G}$. In the PD regime, retrapping processes cause a progressive bending of $\Gamma$ and symmetrization of switching hystograms signalled by the thermal dependence of $\gamma(b)$.

fields of $0 \mathrm{G}$ and $12 \mathrm{G}$ respectively. $H=12 \mathrm{G}$ lowers $I_{c 0}$ reducing at the same time the crossover temperature $T_{\mathrm{cr}}$. Relevant device parameters are summarized in Table 1.

Table 1. Device parameters

\begin{tabular}{c|c|c|c|c|c|c}
\hline \hline Sample & Angle, ${ }^{\circ}$ & $I_{c 0}, \mu \mathrm{A}$ & $J_{c}, \mathrm{~A} / \mathrm{cm}^{2}$ & $C, \mathrm{fF}$ & $Q$ & $T_{\text {cr }}, \mathrm{mK}$ \\
\hline \hline $\mathrm{A}$ & 50 & 1.79 & $5 \cdot 10^{2}$ & 74 & 1.28 & 144 \\
$\mathrm{~B}$ & 75 & 1.20 & $5 \cdot 10^{2}$ & 64 & 1.30 & 135 \\
\hline \hline
\end{tabular}

Taking advantage of the design flexibility of state-ofthe-art $\mathrm{YBaCuO}$ GB biepitaxial JJs $[1,34]$, we have sub- 


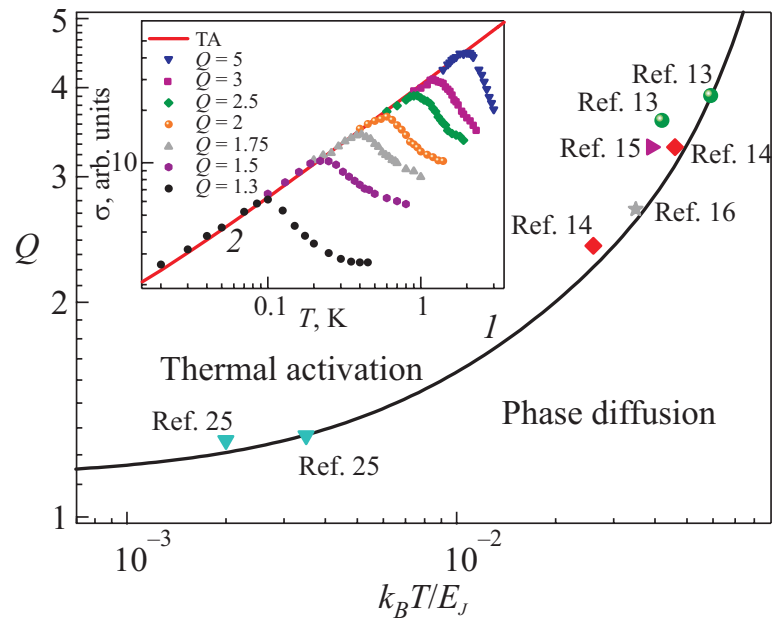

Fig. 4. (Color online) $\left(Q, k_{B} T / E_{J}\right)$ phase diagram. The black line (1) indicates the transition curve between TA and PD regime, which has been extrapolated through Monte Carlo simulations. The points refer to various works reported in literature. The good agreement between experiments and simulations demonstrates the universal character of the phase diagram. In the inset, thermal behavior of $\sigma$ calculated through numerical simulations based on different values of $Q$ is shown. The red line (2) indicates the canonical thermal behavior of $\sigma$. The transition temperature $T^{*}$ and the shape of $\sigma$ in the PD regime are both affected by the junction quality factor $Q$.

stantially engineered devices with $T^{*}<T_{\text {cr }}$ [25]. For temperatures $T$ well below $T_{\mathrm{cr}}$, MQT contributions to escape rates are larger than those coming from both thermal escape and multiple retrapping processes differently from the case $T>T_{\mathrm{cr}}$. In the PD regime the level of dissipation is such that, after an escape event, the phase can be retrapped in a subsequent valley and therefore does not simply roll down the washboard potential. Retrapping processes affect the symmetry of the SCDs. The parameter which is commonly used to measure the symmetry of a generic distribution is the skewness $\gamma[16,20]$. This is defined as the ratio $m_{3} / \sigma^{3}$ where $m_{3}$ is the third central moment of the distribution. $\gamma=0$ corresponds to a symmetric distribution while an asymmetric distribution with a tail on the left (right) side has $\gamma<0 \quad(\gamma>0)$. Phase diffusion also appears in the escape rates $\Gamma$, shown in Fig. 3(b), as a function of the switching current $I$. The escape rates are calculated from the switching distributions using Eq. 5. Below $T_{\mathrm{cr}}$, the escape rates approximately fall on a straight line according to MQT escape rate (Eq. 3), while above $T_{\text {cr }}$ retrapping processes cause a bending in $\Gamma$ versus $I$ curves $[14,16]$. In the PD regime the switching distributions become more symmetric as signalled by the dependence of $\gamma$ on the temperature (see inset of Fig. 3(b)).

Monte Carlo simulations have been performed for different values of the quality factor $Q$ ranging from 1 to 5 . Only TA and PD processes have been calculated in the graphs reported in the inset of Fig. 4. For each of these curves, $T^{*}$ approximately indicates the transition temperature from TA to the diffusive regime. $Q$ tunes $T^{*}$ and modifies the slope of the $\sigma(T)$ fall-off at higher temperatures. Such numerical simulations also allow us to fully reconstruct the $\left(Q, k_{B} T / E_{J}\right)$ diagram reported in Fig. 4 $[13,25]$. The transition curve between the PD regime and the running state following TA has been determined numerically by varying the quality factor $Q$ as function of the ratio between the thermal energy and the Josephson energy. In Fig. $4 Q$ and the ratio $k_{B} T / E_{J}$ are derived for all experiments pointing to PD both on LTS and HTS JJs [13-16,25] and compared with numerical outcomes. The proximity of experimental data to the simulations suggests the validity of such approach and the 'universal' nature of the transition curve. A phase diagram valid in a large range of dissipation conditions emerges as a functional guide to classify the switching behavior and to settle the fundamental junction parameters and energies in the MDR. It is therefore a reference for phase dynamics of novel types of junction and system for which the nature of the currentinduced transition from the superconducting to the normal state has not been completely clarified.

\section{Conclusions}

In summary, we have observed MQT in the moderately damped regime in $\mathrm{GB} \mathrm{YBaCuO}$ JJs and a direct transition from the quantum activation regime to the phase diffusive regime. The diffusive regime appears to be of higher importance with the advent of JJs of very reduced size which are becoming to be highly utilized in superconducting hybrid nanoscale devices $[5,6,36,37]$. This experiments contributes to settle an unexplored region of the phase diagram which is a valid guideline in order to define the relation between the fundamental junction parameters and energies in moderately damped systems.

\section{Acknowledgments}

This work is supported by MIUR PRIN 2009 under the project "SuFET based on nanowires and HTS". We also acknowledge partial support by a Marie Curie International Reintegration Grant No. 248933 hybMQC within the 7th European Community Framework Programme and by "FIRB-Futuro in Ricerca 2012" — Project HybridNanoDev (Grant No. RBFR1236VV).

1. T. Bauch, F. Lombardi, F. Tafuri, A. Barone, G. Rotoli, P. Delsing, and T. Claeson, Phys. Rev. Lett. 94, 087003 (2005); T. Bauch, T. Lindstrom, F. Tafuri, G. Rotoli, P. Delsing, T. Claeson, and F. Lombardi, Science 311, 56 (2006).

2. K. Inomata, S. Sato, K. Nakajima, A. Tanaka, Y. Takano, H.B. Wang, M. Nagao, H. Hatano, and S. Kawabata, Phys. Rev. Lett. 95, 107005 (2005).

3. X.Y. Jin, J. Lisenfeld, Y. Koval, A. Lukashenko, A.V. Ustinov, and P. Mueller, Phys. Rev. Lett. 96, 177003 (2006). 
4. G.-H. Lee, D. Jeong, J.-H. Choi, Y.-J. Doh, and H.-J. Lee, Phys. Rev. Lett. 107, 146605 (2011).

5. M. Sahu, M.H. Bae, A. Rogachev, D. Pekker, T.C. Wei, N. Shah, P.M. Goldbart, and A. Bezryadin, Nat. Phys. 5, 503 (2009).

6. P. Li, P.M. Wu, Y. Bomze, I.V. Borzenets, G. Finkelstein, and A.M. Chang, Phys. Rev. Lett. 107, 137004 (2011).

7. J. Clarke and F.K. Wilhelm, Nature (London) 453, 1031 (2008).

8. C.C. Tsuei and J.R. Kirtley, Rev. Mod. Phys. 72, 969 (2000).

9. F. Tafuri and J.R. Kirtley, Rep. Progr. Phys. 68, 2573 (2005).

10. F. Lombardi, F. Tafuri, F. Ricci, F. Miletto Granozio, A. Barone, G. Testa, E. Sarnelli, J.R. Kirtley, and C.C. Tsuei, Phys. Rev. Lett. 89, 207001 (2002).

11. R.L. Kautz and J.M. Martinis, Phys. Rev. B 42, 9903 (1990); J.M. Martinis and R.L. Kautz, Phys. Rev. Lett. 63, 1507 (1989).

12. M. Iansiti, A.T. Johnson, W.F. Smith, H. Rogalla, C.J. Lobb, and M. Tinkham, Phys. Rev. Lett. 59, 489 (1987); M. Iansiti, M. Tinkham, A.T. Johnson, W.F. Smith, and C.J. Lobb, Phys. Rev. B39, 6465 (1989).

13. J.M. Kivioja, T.E. Nieminen, J. Claudon, O. Buisson, F.W.J. Hekking, and J.P. Pekola, Phys. Rev. Lett. 94, 247002 (2005).

14. J. Männik, S. Li, W. Qiu, W. Chen, V. Patel, S. Han, and J.E. Lukens, Phys. Rev. B 71, 220509 (2005).

15. H.F. Yu, X.B. Zhu, Z.H. Peng, Ye Tian, D.J. Cui, G.H. Chen, D.N. Zheng, X.N. Jing, Li Lu, S.P. Zhao, and S. Han, Phys. Rev. Lett. 107, 067004 (2011).

16. L. Longobardi, D. Massarotti, G. Rotoli, D. Stornaiuolo, G. Papari, A. Kawakami, G.P. Pepe, A. Barone, and F. Tafuri, Phys. Rev. B 84, 184504 (2011).

17. L. Longobardi, D. Massarotti, G. Rotoli, D. Stornaiuolo, G. Papari, A. Kawakami, G.P. Pepe, A. Barone, and F. Tafuri, Appl. Phys. Lett. 99, 062510 (2011).

18. D. Massarotti, L. Longobardi, L. Galletti, D. Stornaiuolo, D. Montemurro, G.P. Pepe, G. Rotoli, A. Barone, and F. Tafuri, Fiz. Nizk. Temp. 38, 336 (2012) [Low Temp. Phys. 38, 263 (2012)].

19. V.M. Krasnov, T. Bauch, S. Intiso, E. Harfeld, T. Akazaki, H. Takayanagi, and P. Delsing, Phys. Rev. Lett. 95, 157002 (2005); V.M. Krasnov, T. Golod, T. Bauch, P. Delsing, Phys. Rev. B 76, 224517 (2007).

20. J.C. Fenton and P.A. Warburton, Phys. Rev. B 78, 054526 (2008).

21. Myung-Ho Bae, M. Sahu, Hu-Jong Lee, and A. Bezryadin, Phys. Rev. B 79, 104509 (2009).
22. Y.J. Doh, J.A. van Dam, A.L. Roest, E.P.A.M. Bakkers, L.P. Kouwenhoven, and S. De Franceschi, Science 309, 272 (2005).

23. M. Veldhorst, C.G. Molenaar, X.L. Wang, H. Hilgenkamp, and A. Brinkman, Appl. Phys. Lett. 100, 072602 (2012).

24. M. Veldhorst, M. Snelder, M. Hoek, T. Gang, X.L. Wang, V.K. Guduru, U. Zeitler, W.G. Wiel, A.A. Golubov, H. Hilgenkamp, and A. Brinkman, Nat. Mater. 11, 417 (2012).

25. L. Longobardi, D. Massarotti, D. Stornaiuolo, L. Galletti, G. Rotoli, F. Lombardi, and F. Tafuri, Phys. Rev. Lett. 109, 050601 (2012).

26. A. Barone and G. Paternò, Physics and Applications of the Josephson Effect, John Wiley and Sons (1982).

27. H.A. Kramers, Physica (Utrecht) 7, 284 (1940).

28. A.O. Caldeira and A.J. Leggett, Phys. Rev. Lett. 46, 211 (1981); A.O. Caldeira and A.J. Leggett, Ann. Phys. 149, 374 (1983).

29. M.H. Devoret, J.M. Martinis, and J. Clarke, Phys. Rev. Lett. 55, 1908 (1985); J.M. Martinis, M.H. Devoret, and J. Clarke, Phys. Rev. B 35, 4682 (1987).

30. W.C. Stewart, Appl. Phys. Lett. 12, 277 (1968); Y.C. Chen, M.P.A. Fisher, and A.J. Leggett, J. Appl. Phys. 64, 6 (1988); M.G. Castellano, G. Torrioli, F. Chiarello, C. Cosmelli, and P. Carelli, J. Appl. Phys. 86, 11 (1999); R. Cristiano and P. Silvestrini, J. Appl. Phys. 60, 9 (1986); B. Ruggiero, V. Corato, C. Granata, L. Longobardi, S. Rombetto, and P. Silvestrini, Phys. Rev. B 67, 132504 (2003).

31. T.A. Fulton and L.N. Dunkleberger, Phys. Rev. B 9, 4760 (1974).

32. Y. Yoon, S. Gasparinetti, M. Mottonen, and J.P. Pekola, J. Low Temp. Phys. 163, 164 (2011).

33. G. Rotoli, T. Bauch, T. Lindstrom, D. Stornaiuolo, F. Tafuri, and F. Lombardi, Phys. Rev. B 75, 144501 (2007).

34. D. Stornaiuolo, G. Papari, N. Cennamo, F. Carillo, L. Longobardi, D. Massarotti, A. Barone, and F. Tafuri, Supercond. Sci. Technol. 24, 045008 (2011).

35. D. Stornaiuolo, G. Rotoli, K. Cedergren, D. Born, T. Bauch, F. Lombardi, and F. Tafuri, J. Appl. Phys. 107, 11390 (2010)

36. P. Lucignano, A. Mezzacapo, F. Tafuri, and A. Tagliacozzo, Phys. Rev. B 86, 144513 (2012).

37. F. Tafuri, D. Massarotti, L. Galletti, D. Stornaiuolo, D. Montemurro, L. Longobardi, P. Lucignano, G. Rotoli, G.P. Pepe, A. Tagliacozzo, and F. Lombardi, J. Supercond. Nov. Magn. 26, 21 (2013). 